\title{
Overview: a fishy story - the roles of rods and nets in maintaining representative longitudinal survey samples
}

\author{
Peter Lynn \\ University of Essex, Institute for Social and Economic Research \\ plynn@essex.ac.uk
}

If you talk to a keen angler, he or she will be replete with stories about favourite locations for angling, special bait, or a particularly effective way of casting; in short, those aspects of their craft that increase the probability of a catch. With a modest stretch of the imagination, angling can be thought of as an analogy for gaining survey co-operation. Survey researchers and interviewers are replete with their own stock of practices that they believe increase the probability of a sample member taking part in their survey. Both the angling enthusiast and the survey researcher may be able to draw on some evidence regarding the extent to which particular methods tend to be successful and the mechanisms through which this success is achieved. But both will also be influenced by subjective personal preferences and beliefs, often with little or no basis in evidence.

The angling enthusiast is unlikely, however, to regale too many stories about how to avoid his or her fish escaping once it has been caught. There will be the occasional tale of a particularly wily fish leaping out of the keepnet to freedom, but by and large the problem of how to keep hold of a fish once caught has been nailed. The same is not true of the survey researcher. The problem of how to keep sample members in a longitudinal study is a very real and worrisome one, which is often debated in survey organisations, amongst survey funders, and at scientific workshops and conferences.

Survey researchers have for many years been setting about the task of obtaining evidence regarding how and why a variety of factors might affect their chances of making a catch, i.e. getting a selected sample member to participate in their survey. But only in the last couple of decades has considerable attention been paid to the factors that might affect the probability of keeping hold of that initial catch, i.e. avoiding sample attrition (Campanelli and O'Muircheartaigh 1999, Fitzgerald et al 1998, Laurie et al 1999, Lepkowski and Couper 2002). In the UK, the importance of sample attrition was recognised by a special conference held in May 2004 (Lynn 2006) and by the Survey Design and Measurement Initiative (SDMI) of the Economic and Social Research Council (Lynn and Erens 2010). Two of the six research projects commissioned in 2007 under SDMI were specifically focussed on attrition, while a third addressed non-response more generally but with a sub-project focussed on attrition. The importance of research into ways of dealing with non-response on longitudinal surveys has never been greater.

There are perhaps two main reasons why longitudinal survey researchers are concerned about sample attrition. The first is simply that a reduction in sample size will reduce the precision of estimates. Ultimately, there may be a fear that the sample size reduces to the extent that funders decide that the study is not worth continued support. The second is that non-response and attrition may introduce bias to estimates. This will happen if non-respondents differ systematically from respondents in terms of the key measures (Watson and Wooden 2009).

This special issue of Longitudinal and Life Course Studies is devoted to non-response and attrition in longitudinal surveys of ageing. All four contributions use data from the (American) Health and Retirement Study (HRS). Three of the four involve comparisons with the English Longitudinal Study of Aging (ELSA). The authors tackle a number of important issues. Banks et al and Cheshire et al both attempt to identify 
and explain differences in response rates between the two surveys. They are both successful at identification but less successful at explanation. The latter result is rather inevitable when you only have two observations (the surveys) and several potential explanatory factors. Nevertheless, the exercise is not fruitless, as both papers highlight important differences between the two surveys and suggest promising avenues for further research.

One of the important differences between HRS and ELSA is examined in the paper by Weir et al, namely the ready acceptance by HRS of a proxy interview in cases where a personal interview with the sample member is not possible. Survey strategy regarding the use of proxy interviews is often discussed but under-researched (Moore 1988). There are trade-offs to be made between overall response and personal (as opposed to proxy) response, particularly on a longitudinal survey where accepting a proxy response at one wave may make it harder to obtain a personal response in the future. This might not matter were there not also a trade-off between non-response error and measurement error. Weir et al show that the acceptance of proxies explains part of the response rate difference between the two surveys but, perhaps more importantly, explains all of the differential bias in terms of cognitive ability. This demonstrates the important role that proxy interviews can play and is a good example of a study that moves beyond looking at effects on response rates and examines effects on the bias of substantive measures of interest.

The paper by Michaud et al also is also concerned with bias in substantive measures, specifically estimates from realistic panel data models. Their interest is in the effect of converting previous wave non-respondents to become current wave respondents. Burton et al (2006) showed that refusal conversion attempts are worthwhile for longitudinal surveys, in the sense that the successfully converted sample members often then remain respondents for many subsequent waves. But that work did not assess the impact on substantive estimates. Michaud et al compare estimates using the full HRS data with those that would be obtained if observations subsequent to a wave non-response were excluded. They conclude that panel model estimates of wealth would be substantially biased if these sample members had not been subsequently converted to become respondents.

Collectively, the papers in this special issue should serve to remind longitudinal researchers that error in their substantive estimates can be influenced by many aspects of survey design and implementation. Decisions about survey procedures - such as whether and in what circumstances to accept or seek proxy responses, or whether and how to seek responses from previous wave non-respondents - make a difference. These decisions do not merely make a difference to response rate; they can also affect bias in estimates. Different decisions may have different implications for bias. This point should certainly be of concern to researchers interested in comparing estimates from surveys that have used different procedures, or indeed researchers drawing upon data from multiple waves of a survey that has changed its procedures over time.

Procedures that matter are not only those relating to the use of proxy respondents and to attempting to interview previous wave nonrespondents. The papers by Banks et al and Cheshire et al also highlight differences between HRS and ELSA in procedures such as the use of respondent incentives, sample design, field issue policy, data collection mode and between-wave contacts. All these and more could potentially introduce differential non-response error and/or differential measurement error. Researchers have recently begun to look beyond the use of procedures that are standardised across the whole sample, and to examine whether procedures tailored to the circumstances of particular subgroups might be more effective in combating attrition. A couple of recent studies have investigated a number of ways in which this might be done (Fumagalli et al 2010, McGonagle et al 2009). This seems like a promising avenue to pursue.

We still have a long way to go to understand the nature and causes of all errors in longitudinal survey data. We should constantly re-assess and re-evaluate all survey features and procedures. And with respect to non-response error, it is both the features of our fishing rod and the features of our keepnet that are important. 


\section{References}

Burton J, Lynn P and Laurie H. (2006) The long-term effectiveness of refusal conversion procedures on longitudinal surveys. Journal of the Royal Statistical Society Series A (Statistics in Society), 169, 459-478.

Campanelli P and O'Muircheartaigh C. (1999) Interviewers, interviewer continuity, and panel survey nonresponse. Quality and Quantity, 33, 59-76.

Fitzgerald J, Gottschalk P and Moffitt R. (1998) An analysis of sample attrition in panel data: The Michigan Panel Study of Income Dynamics. The Journal of Human Resources, 33, 251-299.

Fumagalli L, Laurie H and Lynn P. (2010) Experiments with methods to reduce attrition in longitudinal surveys. ISER Working Paper 2010-04, Colchester: ISER, University of Essex. Downloaded on 3-10-2010 from www.iser.essex.ac.uk/publications/working-papers/iser/2010-04.pdf.

Laurie $\mathrm{H}$, Smith R and Scott L. (1999) Strategies for reducing nonresponse in a longitudinal panel survey. Journal of Official Statistics, 15, 269-282.

Lepkowski J and Couper MP. (2002) Nonresponse in the second wave of longitudinal household surveys. In RM Groves et al. eds. Survey Nonresponse, Wiley, New York.

Lynn P. (2006) Attrition and non-response. Journal of the Royal Statistical Society Series A (Statistics in Society), $169,393-394$.

Lynn P and Erens B. (2010) The ESRC Survey Resources Network: Opportunities for the advancement of survey methods. International Journal of Market Research, 52, 295-302.

McGonagle K, Couper M and Schoeni R. (2009) An experimental text of a strategy to maintain contact with families between waves of a panel study. Survey Practice, June 2009. Downloaded on 3-10-2010 from http://surveypractice.org/2009/06/29/panel-contacts.

Moore JC. (1988) Self/proxy response status and survey response quality, a review of the literature. Journal of Official Statistics, 4, 155-172.

Watson N and Wooden M. (2009) Identifying factors affecting longitudinal survey response. In P Lynn, ed. Methodology of Longitudinal Surveys, Wiley, Chichester. 